\title{
On the Use of Elzaki Decomposition Method for Solving Higher-Order Integro-Differential Equations
}

\author{
Ekhlass S. Al-Rawi (iD) and Merna A. Samarchi \\ College of Computer Sciences and Mathematics, University of Mosul, Mosul, Iraq \\ Correspondence should be addressed to Ekhlass S. Al-Rawi; drekhlass-alrawi@uomosul.edu.iq
}

Received 6 December 2021; Accepted 19 January 2022; Published 21 February 2022

Academic Editor: Shyam L. Kalla

Copyright () 2022 Ekhlass S. Al-Rawi and Merna A. Samarchi. This is an open access article distributed under the Creative Commons Attribution License, which permits unrestricted use, distribution, and reproduction in any medium, provided the original work is properly cited.

In this article, we implemented the Elzaki decomposition technique (EDM) to solve Volterra-Fredholm integro-differential equations of higher-order. Illustrations are used to test the technique's accuracy and validity. Comparison among the acquired consequences by EDM and actual solutions have proven the power and accuracy of this technique. This technique is dependable and able to supply analytic remedies for solving such equations.

\section{Introduction}

This study is concerned with important problems in the sciences of engineering, which contain both integral and differential factors. In the early 20th century, this kind of equation was first presented by Volterra, where he studied genetic influences on population growth by developing the topic of integral and differential equations [1].

Many researchers, engineers, and scientists face problems of an electrical circuit and biological species when solving differential equations resulting from the spread of heat and mass that appear with increasing and decreasing generation rates.

In physics, engineering, and biology applications, one can find additional details about the origins of these equations as well as the behavior of advanced integral equations [2]. The Taylor collocation, Adomian decomposition, Chebyshev, Haar wavelet, successive approximation, Bernstein, homotopy perturbation, and other technologies are widely used to work with the IDEs of higher-order [3-13]. In recent years, Elzaki transform (ET) was introduced by Tarig Elzaki where it was used to solve different types of equations with integral, differential, partial integral, integro-differential, and fractional-order equations [14-20].

The motivation of the article is to enhance the analysis of the modified Elzaki transformation, known as the Elzaki decomposition method (EDM), to solve the higher-order IDE of Volterra-Fredholm of the form

$$
\begin{aligned}
y^{(n)}(z)= & f(z)+\lambda_{1} \int_{a}^{z} k_{1}(z, \tau) W_{1}(y(\tau)) d \tau \\
& +\lambda_{2} \int_{a}^{b} k_{2}(z, \tau) W_{2}(y(\tau)) d \tau,
\end{aligned}
$$

subject to

$$
\begin{aligned}
y^{(m)}(a) & =y_{m}, \\
m & =0,1,2, \ldots, n-1,
\end{aligned}
$$

where $a, b, \lambda_{1}, \lambda_{2}, y_{m}$ are constant values, $f(z), k_{1}(z, \tau), k_{2}(z, \tau)$ are given forms with appropriate interval derivatives $a \leq z \leq \tau \leq b, W_{1}(y(\tau))$, and $W_{2}(y(\tau))$ are nonlinear functions in $y(\tau)$.

\section{Elzaki Transformation Method (ETM) with Their Properties}

The Elzaki transform (ET) of $f(\tau) \in \Re, \forall \tau \geq 0$ as in [21].

$$
E[f(\tau)]=F(\omega)=\omega \int_{0}^{\infty} f(\tau) e^{(-\tau / \omega)} d \tau \quad h_{1} \leq \omega \leq h_{2} .
$$


Since $E[$.$] is the operator of Elzaki transform and the set$ $\mathfrak{R}$ is defined as

$$
\begin{aligned}
\Re= & \left\{f(\tau)\left|\exists C, h_{1}, h_{2}>0,\right| f(\tau) \mid<C e^{\tau / h_{n}},\right. \\
& \text { if } \left.\tau \in(-1)^{n} \times[0, \infty)\right\},
\end{aligned}
$$

$h_{1}, h_{2}$ can be finite or infinite, and $C$ is a real finite number.

If $E[f(\tau)]=F(\omega)$, then $f(\tau)$ is called the inverse transform of Elzaki to $F(\omega)$ and denoted by $f(\tau)=E^{-1}[F(\omega)]$, where $E^{-1}$ is the operator of inverse Elzaki transform. Table 1 shows the transformation of Elzaki for some basic functions [22].

Special properties of the Elzaki transform are as follows.

Theorem 1 (linearity, see [23]). If $F_{1}(\omega)$ and $F_{2}(\omega)$ are Elzaki transform $(E T)$ of $f_{1}(\tau)$ and $f_{2}(\tau)$, respectively, then

$$
\begin{aligned}
& E\left\{\alpha f_{1}(\tau)+\beta f_{2}(\tau)\right\} \\
& \quad=\alpha E\left\{f_{1}(\tau)\right\}+\beta E\left\{f_{2}(\tau)\right\} \\
& =\alpha F_{1}(\omega)+\beta F_{2}(\omega) .
\end{aligned}
$$

$\alpha, \beta$ are constants.

Theorem 2 (differentiation, see [21]). If the transform of Elzaki for $f(\tau)$ given by $E[f(\tau)]=F(\omega)$, then

$$
\begin{aligned}
E\left[f^{\prime(\tau)}\right] & =\frac{F(\omega)}{\omega}-\omega f(0), \\
E\left[f^{\prime \prime}(\tau)\right] & =\frac{F(\omega)}{\omega^{2}}-f(0)-\omega f^{\prime}(0), \\
E\left[f^{(n)}(\tau)\right] & =\frac{F(\omega)}{\omega^{n}}-\sum_{k=0}^{n-1} \omega^{2-n+k} f^{(k)}(0) .
\end{aligned}
$$

Theorem 3 (shift, see [23]). If $f(\tau) \in \mathfrak{R}$ with Elzaki transform $F(\omega)$, then

$$
E\left[e^{a \tau} f(\tau)\right]=\frac{1}{1-a \omega} F\left[\frac{\omega}{1-a \omega}\right] .
$$

Theorem 4 (convolution theorem, see [23]).

If $f_{1}(\tau)$ and $f_{2}(\tau)$ are functions with Elzaki transform $F_{1}(\omega)$ and $F_{2}(\omega)$, then the convolution of $f_{1}$ and $f_{2}$ is
TABLE 1: Special functions by transform of Elzaki.

\begin{tabular}{lc}
\hline$f(\tau)$ & $E[f(\tau)]=F(\omega)$ \\
\hline 1 & $\omega^{2}$ \\
$\tau$ & $\omega^{3}$ \\
$\tau^{2}$ & $2 ! \omega^{4}$ \\
$\tau^{n}, n \in N$ & $n ! \omega^{n+2}$ \\
$e^{a \tau}$ & $\omega^{2} / 1-a \omega$ \\
$\sin a \tau$ & $a \omega^{3} / 1+a^{2} \omega^{2}$ \\
$\cos a \tau$ & $\omega^{2} / 1+a^{2} \omega^{2}$ \\
$\sinh a \tau$ & $a \omega^{3} / 1-a^{2} \omega^{2}$ \\
$\cosh a \tau$ & $\omega^{2} / 1-a^{2} \omega^{2}$ \\
$\tau e^{a \tau}$ & $\omega^{3} /(1-a \omega)^{2}$ \\
\hline
\end{tabular}

$$
\left(f_{1} * f_{2}\right)(\tau)=\int_{0}^{\tau} f_{1}(x) f_{2}(\tau-x) \mathrm{d} x .
$$

Also, Elzaki transform is

$$
E\left[f_{1} * f_{2}\right]=\frac{1}{\omega} F_{1}(\omega) F_{2}(\omega)
$$

Theorem 5 (integration, see [23]). Let $F(\omega)$ denote to the transform of Elzaki for $f(\tau)$, then the definite integral of $f(\tau)$,

$$
\mathrm{L}(\tau)=\int_{0}^{\tau} f(\tau) d \tau
$$

has transform of Elzaki as

$$
E[L(\tau)]=\omega F(\omega)
$$

\section{The Proposed Technique (EDM)}

The proposed technique of the method (EDM) is as follows: we suppose that the (1) and (2) have sufficiently differentiable and unique solution $y(z)$. We use the Elzaki decomposition method to get an approximation for the integro-differential equations of high order.

Applying the ETM of both sides of (1), we get

$$
\begin{aligned}
E\left[y^{(n)}(z)\right]= & E[f(z)]+E\left[\lambda_{1} \int_{a}^{z} k_{1}(z, \tau) W_{1}(y(\tau)) d \tau\right] \\
& +E\left[\lambda_{2} \int_{a}^{b} k_{2}(z, \tau) W_{2}(y(\tau)) d \tau\right] .
\end{aligned}
$$

Using Theorem 2, we get

$$
\frac{E[y(z)]}{\omega^{n}}-\sum_{k=0}^{n-1} \omega^{2-n+k} y^{(k)}(0)=E[f(z)]+E\left[\lambda_{1} \int_{a}^{z} k_{1}(z, \tau) W_{1}(y(\tau)) d \tau\right]+E\left[\lambda_{2} \int_{a}^{b} k_{2}(z, \tau) W_{2}(y(\tau)) d \tau\right]
$$


Solving for $E[y(z)]$, we get

$E[y(z)]=\omega^{n} \sum_{k=0}^{n-1} \omega^{2-n+k} y^{(k)}(0)+\omega^{n} E[f(z)]+\omega^{n} E\left[\lambda_{1} \int_{a}^{z} k_{1}(z, \tau) W_{1}(y(\tau)) d \tau\right]+\omega^{n} E\left[\lambda_{2} \int_{a}^{b} k_{2}(z, \tau) W_{2}(y(\tau)) d \tau\right]$.

Now, the EDM represents the solution $y(z)$ of our problem as infinite series

$$
y(z)=\sum_{j=0}^{\infty} y_{j}(z)
$$

Using the recursive relationship, the components $E$ [ $y$ $(z)$ ] will be obtained, and the polynomials of the infinite series will be substituted for the nonlinear functions $W_{1}(y)$ and $W_{2}(y)$.

$$
\begin{aligned}
& W_{1}(y)=\sum_{j=0}^{\infty} A_{j}, \\
& W_{2}(y)=\sum_{j=0}^{\infty} B_{j},
\end{aligned}
$$

where $A_{j}$ and $B_{j}$ are the Adomian polynomials that are created using Adomian's specialized algorithms [24].

Substituting equations (15) and (16) in (14), we have

$$
E\left[\sum_{j=0}^{\infty} y_{j}(z)\right]=\omega^{n} \sum_{k=0}^{n-1} \omega^{2-n+k} y^{(k)}(0)+\omega^{n} E[f(z)]+\omega^{n} E\left[\lambda_{1} \int_{a}^{z} k_{1}(z, \tau) \sum_{j=0}^{\infty} A_{j} d \tau\right]+\omega^{n} E\left[\lambda_{2} \int_{a}^{b} k_{2}(z, \tau) \sum_{j=0}^{\infty} B_{j} d \tau\right] .
$$

Now,

$$
E\left[y_{0}(z)\right]=\omega^{n} \sum_{k=0}^{n-1} \omega^{2-n+k} y^{(k)}(0)+\omega^{n} E[f(z)] .
$$

The rest components of $\mathrm{E}\left[\mathrm{y}_{\mathrm{j}}(\mathrm{z})\right]$ can be found using the previous solutions as

$$
\begin{aligned}
E\left[y_{j+1}(z)\right]= & \omega^{n} E\left[\lambda_{1} \int_{a}^{z} k_{1}(z, \tau) \sum_{j=0}^{\infty} A_{j} d \tau\right] \\
& +\omega^{n} E\left[\lambda_{2} \int_{a}^{b} k_{2}(z, \tau) \sum_{j=0}^{\infty} B_{j} d \tau\right] .
\end{aligned}
$$

As a result, the components $y_{0}, y_{1}, y_{2}, \ldots$ are identified by applying the inverse Elzaki transform of (18) and (19) to obtain

$$
\begin{aligned}
y_{0}(z)= & E^{-1}\left[\omega^{n} \sum_{k=0}^{n-1} \omega^{2-n+k} y^{(k)}(0)\right]+E^{-1}\left[\omega^{n} E[f(z)]\right], \\
y_{j+1}(z)= & E^{-1}\left[\omega^{n} E\left[\lambda_{1} \int_{a}^{z} k_{1}(z, \tau) \sum_{j=0}^{\infty} A_{j} d \tau\right]\right] \\
& +E^{-1}\left[\omega^{n} E\left[\lambda_{2} \int_{a}^{b} k_{2}(z, \tau) \sum_{j=0}^{\infty} B_{j} d \tau\right]\right], \quad j \geq 0 .
\end{aligned}
$$

\section{Numerical Examples}

To apply the proposed technique (EDM), various examples of higher orders were taken, and to study the efficiency of the suggested technique, a comparison with the exact solution was made. The calculations of solutions for examples are performed using the Maple program.

Example 1. The third order linear IDE of VolterraFredholm is

$$
y^{\prime \prime \prime}(z)=-\frac{1}{2} z^{2}+\int_{0}^{z} y(\tau) d \tau+\int_{-\pi}^{\pi} z y(\tau) d \tau, \quad 0 \leq z \leq 1,
$$

subject to

$$
y(0)=1, y^{\prime}(0)=1, y^{\prime \prime}(0)=-1 .
$$

Applying to both sides of (21) Elzaki transform, we get $E\left[y^{\prime \prime \prime}(z)\right]=E\left[-\frac{1}{2} z^{2}\right]+E\left[\int_{0}^{z} y(\tau) d \tau\right]+E\left[\int_{-\pi}^{\pi} z y(\tau) d \tau\right]$.

We use the initial conditions together with Theorems 2 and 5 to obtain

$$
\frac{E[y(z)]}{\omega^{3}}=1+\frac{1}{\omega}-\omega-\omega^{4}+\omega E[y(z)]+\omega^{3} \int_{-\pi}^{\pi} y(\tau) d \tau .
$$

Solving the (15) for $E[y(z)]$, we get

$$
\begin{aligned}
E[y(z)]= & \frac{\omega^{2}}{1-\omega^{4}}+\frac{\omega^{3}}{1-\omega^{4}}-\frac{\omega^{4}}{1-\omega^{4}}-\frac{\omega^{7}}{1-\omega^{4}} \\
& +\frac{\omega^{6}}{1-\omega^{4}} \int_{-\pi}^{\pi} y(\tau) d \tau .
\end{aligned}
$$

Substituting the series assumptions for $E[y(s)]$ as given in (15) and using the recursive relation, we get 


$$
\begin{aligned}
E\left[y_{0}(z)\right] & =\frac{\omega^{2}}{1-\omega^{4}}+\frac{\omega^{3}}{1-\omega^{4}}-\frac{\omega^{4}}{1-\omega^{4}}-\frac{\omega^{7}}{1-\omega^{4}} E\left[y_{j+1}(z)\right] \\
& =\frac{\omega^{6}}{1-\omega^{4}} \int_{-\pi}^{\pi} y_{j}(\tau) d \tau, \quad j=0,1,2, \ldots
\end{aligned}
$$

Taking the inverse transform of (26), we have

$$
y_{0}(z)=z+\cos z \text {, }
$$

$$
y_{1}(z)=E^{-1}\left[\frac{\omega^{6}}{1-\omega^{4}} \int_{-\pi}^{\pi} y_{0}(\tau) d \tau\right]=0,
$$

$y_{j+1}(z)=E^{-1}\left[\frac{\omega^{6}}{1-\omega^{4}} \int_{-\pi}^{\pi} y_{j}(\tau) d \tau\right]=0, \quad \forall j=2,3, \ldots$

So, the series solution will be the actual solution $y(z)=z+\cos z$.
Example 2. The fourth-order nonlinear IDE of VolterraFredholm is

$$
\begin{aligned}
y^{(4)}(z)= & -\frac{z^{6}}{30}-\frac{z^{4}}{6}-\frac{z^{2}}{2}+\frac{8}{3} z+\frac{3}{2}+\int_{0}^{z}(z-\tau) y^{2}(\tau) d \tau \\
& -2 \int_{0}^{1}(z+\tau) y(\tau) d \tau, \quad 0 \leq z \leq 1,
\end{aligned}
$$

subject to

$$
\begin{gathered}
y(0)=1, \\
y^{\prime}(0)=0, \\
y^{\prime \prime}(0)=2, \\
y^{\prime \prime \prime}(0)=0 .
\end{gathered}
$$

Taking the transformation of Elzaki to (28), we have

$$
E\left[y^{(4)}(z)\right]=E\left[-\frac{z^{6}}{30}\right]-E\left[\frac{z^{4}}{6}\right]-E\left[\frac{z^{2}}{2}\right]+E\left[\frac{8}{3} z\right]+E\left[\frac{3}{2}\right]+E\left[\int_{0}^{z}(z-\tau) y^{2}(\tau) d \tau\right]-E\left[2 \int_{0}^{1}(z+\tau) y(\tau) d \tau\right]
$$

Applying the initial conditions together with Theorems $2-5$, we obtained

$$
\begin{aligned}
\frac{E[y(z)]}{\omega^{4}}= & \frac{1}{\omega^{2}}+2-24 \omega^{8}-4 \omega^{6}-\omega^{4}+\frac{8}{3} \omega^{3}+\frac{3}{2} \omega^{2} \\
& +\omega^{2} E\left[y^{2}(\tau)\right]-2 E\left[\int_{0}^{1}(z+\tau) y(\tau) d \tau\right] .
\end{aligned}
$$

Now, solving the (31) for $E[y(z)]$, we get

$$
\begin{aligned}
E[y(z)]= & \omega^{2}+2 \omega^{4}-24 \omega^{12}-4 \omega^{10}-\omega^{8}+\frac{8}{3} \omega^{7}+\frac{3}{2} \omega^{6} \\
& +\omega^{6} E\left[y^{2}(\tau)\right]-2 \omega^{4} E\left[\int_{0}^{1}(z+\tau) y(\tau) d \tau\right] .
\end{aligned}
$$

Substituting the series assumptions for $E[y(z)]$ as given in (15) and using the recursive relation, we get

$$
\begin{gathered}
E\left[y_{0}(z)\right]=\omega^{2}+2 \omega^{4}-24 \omega^{12}-4 \omega^{10}-\omega^{8}+\frac{8}{3} \omega^{7}+\frac{3}{2} \omega^{6}, \\
E\left[y_{j+1}(z)\right]=\omega^{6} E\left[A_{j}(\tau)\right]-2 \omega^{4} E\left[\int_{0}^{1}(z+\tau) y_{j}(\tau) d \tau\right], \quad j \geq 0,
\end{gathered}
$$

where $A_{n}$ 's are given by

$$
\begin{aligned}
A_{0}(\tau) & =y_{0}^{2}, \\
A_{1}(\tau) & =2 y_{0} y_{1}, \\
A_{2}(\tau) & =2 y_{0} y_{2}+y_{1}^{2}, \\
A_{3}(\tau) & =2 y_{0} y_{3}+2 y_{1} y_{2} . \\
\vdots &
\end{aligned}
$$

We apply for both sides of (33) the inverse Elzaki transform; we obtain general solution given by recursive relation as

$$
\begin{gathered}
y_{0}(z)=E^{-1}\left[\omega^{2}+2 \omega^{4}-24 \omega^{12}-4 \omega^{10}-\omega^{8}+\frac{8}{3} \omega^{7}+\frac{3}{2} \omega^{6}\right], \\
y_{j+1}(z)=E^{-1}\left[\omega^{6} E\left[A_{j}(\tau)\right]\right]-2 E^{-1}\left[\omega^{4} E\left[\int_{0}^{1}(z+\tau) y_{j}(\tau) d \tau\right]\right], \quad j \geq 0,
\end{gathered}
$$


which leads to

$$
\begin{aligned}
y_{0}(z)= & 1+z^{2}+\frac{z^{4}}{16}+\frac{z^{5}}{45}-\frac{z^{6}}{720}-\frac{z^{8}}{10080}-\frac{z^{10}}{151200} \\
y_{1}(z)= & \frac{-73}{1152} z^{4}-\frac{323}{14400} z^{5}+\frac{z^{6}}{720}+\frac{z^{8}}{10080}-\frac{z^{9}}{136080}-\frac{z^{10}}{907200} \\
& +\frac{29}{59875200} z^{11}+\frac{23}{119750400} z^{12}+\frac{37}{778377600} z^{13} \\
& +\frac{41}{58118860800} z^{14}+\frac{1}{851350500} z^{15}+\frac{197}{10461394944000} z^{16} \\
& -\frac{1}{144353664000} z^{17}-\frac{43}{24251415552000} z^{18}-\frac{1}{4430547072000} z^{19} \\
& -\frac{1}{50634823680000} z^{20}-\frac{1}{488663280000} z^{21}-\frac{43}{81876509890560000} z^{22} \\
& +\frac{1}{73849401077760000} z^{24}+\frac{1}{3789640318464000000} z^{26}
\end{aligned}
$$

and so on.

Now, the solution for the (28) will be the following:

$$
y_{\mathrm{EDM}}(z)=\sum_{j=0}^{2} y_{j}(z)=y_{0}(z)+y_{1}(z)+y_{2}(z) \cong 1+z^{2},
$$

which is the actual solution.

Table 2 indicates the evaluation of the numerical results and the exact solution, where the numerical solution exactly corresponds to the exact values as shown in Figure 1.

Example 3. The fifth order nonlinear IDE of VolterraFredholm is

$$
\begin{array}{r}
y^{(5)}(z)=-z e^{3 z}+\int_{0}^{z} e^{3 z-3 \tau} y^{3}(\tau) d \tau+\int_{0}^{1} e^{z-\tau} y(\tau) d \tau, \\
0 \leq z \leq 1,
\end{array}
$$

where

$$
\begin{aligned}
y(0) & =y^{\prime}(0) \\
& =y^{\prime \prime}(0) \\
& =y^{\prime \prime \prime}(0) \\
& =y^{(4)}(0) \\
& =1 .
\end{aligned}
$$

To both sides of (38), we take the Elzaki transform and we get

$$
\begin{aligned}
E\left[y^{(5)}(z)\right]= & E\left[-z e^{3 z}\right]+E\left[\int_{0}^{z} e^{3 z-3 \tau} y^{3}(\tau) d \tau\right] \\
& +E\left[\int_{0}^{1} e^{z-\tau} y(\tau) d \tau\right] .
\end{aligned}
$$

Applying the initial conditions together with Theorems $2-5$, we obtained

$$
\begin{aligned}
\frac{E[y(z)]}{\omega^{5}}= & \frac{1}{\omega^{3}}+\frac{1}{\omega^{2}}+\frac{1}{\omega}+1+\omega-\frac{\omega^{3}}{(1-3 \omega)^{2}} \\
& +\frac{\omega}{1-3 \omega} E\left[y^{3}(\tau)\right]+E\left[\int_{0}^{1} e^{z-\tau} y(\tau) d \tau\right] .
\end{aligned}
$$

Solving the (41) for $E[y(z)]$, we get

$$
\begin{aligned}
E[y(z)]= & \omega^{2}+\omega^{3}+\omega^{4}+\omega^{5}+\omega^{6}-\frac{\omega^{8}}{(1-3 \omega)^{2}} \\
& +\frac{\omega^{6}}{1-3 \omega} E\left[y^{3}(\tau)\right]+\omega^{5} E\left[\int_{0}^{1} e^{z-\tau} y(\tau) d \tau\right] .
\end{aligned}
$$

Substituting the series assumptions for $E[y(z)]$ as given in (15) and using the recursive relation, we get

$$
\begin{aligned}
E\left[y_{0}(z)\right]= & \omega^{2}+\omega^{3}+\omega^{4}+\omega^{5}+\omega^{6}, \\
E\left[y_{j+1}(z)\right]= & -\frac{\omega^{8}}{(1-3 \omega)^{2}}+\frac{\omega^{6}}{1-3 \omega} E\left[A_{j}(t)\right] \\
& +\omega^{5} E\left[\int_{0}^{1} e^{z-\tau} y_{j}(\tau) d \tau\right], \quad j \geq 0,
\end{aligned}
$$


TABLE 2: The approximation numerical $y_{\mathrm{EDM}}$ and $y_{\text {exact }}$ for Example 2.

\begin{tabular}{lccr}
\hline$Z$ & yexact $_{\text {en }}$ & $\mathbf{y}_{\text {E DM }}$ & Absolute error \\
\hline 0 & 1 & 1 & 0 \\
0.1 & 1.01 & 1.009999911111103 & $8.888889690972235 e-08$ \\
0.2 & 1.04 & 1.039998544439903 & $1.455560097562980 e-06$ \\
0.3 & 1.09 & 1.089992462310762 & $7.537689237890888 e-06$ \\
0.4 & 1.16 & 1.159975641732821 & $2.435826717883671 e-05$ \\
0.5 & 1.25 & 1.249939214512404 & $6.078548759624880 e-05$ \\
0.6 & 1.36 & 1.359871181525310 & $1.288184746899290 e-04$ \\
0.7 & 1.49 & 1.489756063481625 & $2.439365183752873 e-04$ \\
0.8 & 1.64 & 1.639574420447577 & $4.255795524235051 e-04$ \\
0.9 & 1.81 & 1.809302131298738 & $6.978687012617613 e-04$ \\
1 & 2 & 1.998909272272858 & $1.090727727141916 e-03$ \\
\hline
\end{tabular}

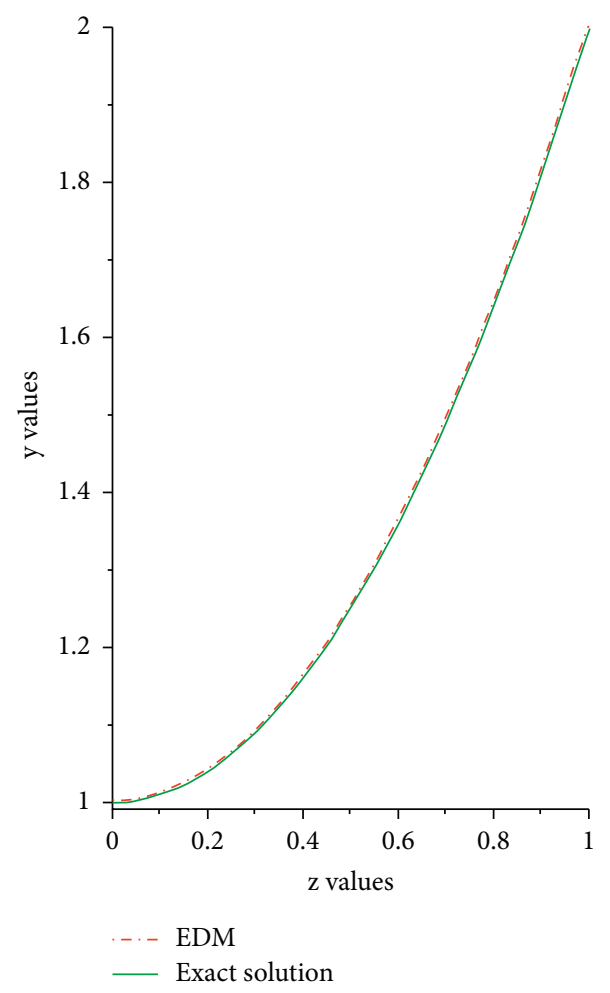

FIgURE 1: The approximate solution $y_{\mathrm{EDM}}$ with $y_{\text {exact }}$ for Example 2.

where $A_{n} / s$ are given by

$$
\begin{aligned}
A_{0}(\tau) & =y_{0}^{3}, \\
A_{1}(\tau) & =3 y_{1} y_{0}^{2} \\
A_{2}(\tau) & =3 y_{2} y_{0}^{2}+3 y_{1}^{2} y_{0}, \\
A_{3}(\tau) & =3 y_{3} y_{0}^{2}+6 y_{0} y_{1} y_{2}+y_{1}^{3} . \\
\vdots &
\end{aligned}
$$

We apply for both sides of (43) the inverse Elzaki transform; we obtain the general solution given by recursive relation as

$$
\begin{aligned}
y_{0}(z)= & E^{-1}\left[\omega^{2}+\omega^{3}+\omega^{4}+\omega^{5}+\omega^{6}\right] \\
y_{j+1}(z)= & -E^{-1}\left[\frac{\omega^{8}}{(1-3 \omega)^{2}}\right]+E^{-1}\left[\frac{\omega^{6}}{1-3 \omega} E\left[A_{j}(t)\right]\right] \\
& +E^{-1}\left[\omega^{5} E\left[\int_{0}^{1} e^{z-\tau} y_{j}(\tau) d \tau\right]\right], \quad j \geq 0,
\end{aligned}
$$

which leads to 


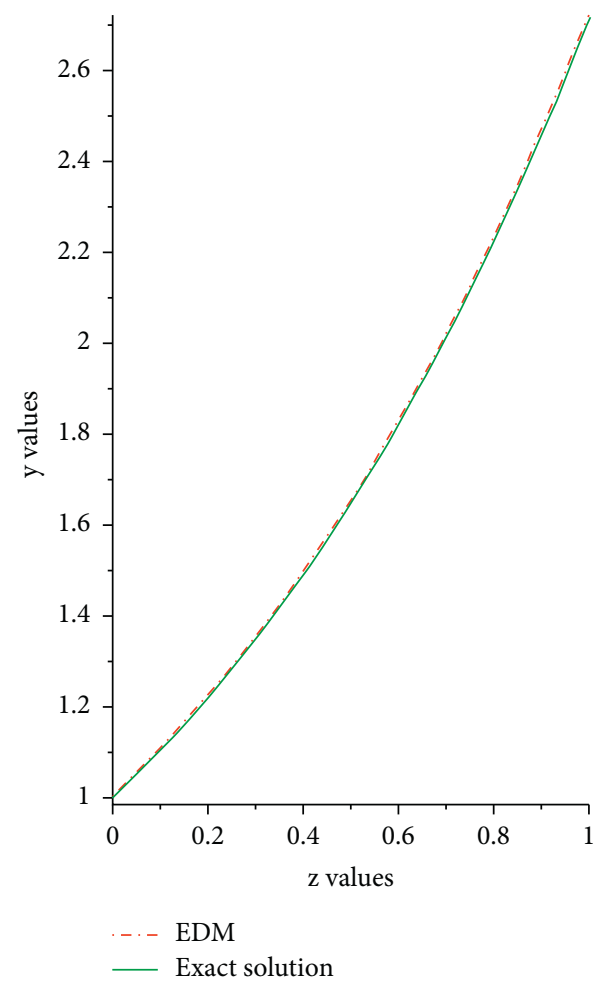

FIGURE 2: Comparing the numerical solution $y_{\mathrm{EDM}}$ with $y_{\text {exact }}$ for Example 3.

TABLE 3: The numerical approximation $y_{\mathrm{EDM}}$ and $y_{\text {exact }}$ for Example 3 .

\begin{tabular}{lccc}
\hline$Z$ & yext $_{\text {eac }}$ & $\mathbf{y}_{\mathrm{E} \text { DM }}$ & Absolute error \\
\hline 0 & 1 & 1 & 0 \\
0.1 & 1.105170918075648 & 1.105170918017268 & $5.837930139307446 e-11$ \\
0.2 & 1.221402758160170 & 1.221402756260010 & $1.900160251366856 e-09$ \\
0.3 & 1.349858807576003 & 1.349858792896531 & $1.467947230437972 e-08$ \\
0.4 & 1.491824697641270 & 1.491824634692902 & $6.294836829567885 e-08$ \\
0.5 & 1.648721270700128 & 1.648721075131608 & $1.955685198229418 e-07$ \\
0.6 & 1.822118800390509 & 1.822118304574017 & $4.958164925294994 e-07$ \\
0.7 & 2.013752707470477 & 2.013751613802701 & $1.093667775897700 e-06$ \\
0.8 & 2.225540928492468 & 2.225538745229276 & $2.183263192012674 e-06$ \\
0.9 & 2.459603111156950 & 2.459599057363046 & $4.053793903668890 e-06$ \\
1 & 2.718281828459046 & 2.718274674142005 & $7.154317040125591 e-06$ \\
\hline
\end{tabular}

$$
\begin{aligned}
y_{0}(z)= & +z+\frac{z^{2}}{2}+\frac{z^{3}}{6}+\frac{z^{4}}{24}, \\
y_{1}(z)= & -\frac{1}{243} z e^{3 z}+\frac{870040}{43046721} e^{3 z}-\frac{3650}{729}-\frac{68}{27} z^{2}-\frac{137}{162} z^{3}-\frac{23}{108} z^{4} \\
& -\frac{1219}{243} z-\frac{87}{8} e^{z-1}+\frac{87}{8} e^{-1}+\frac{87}{8} e^{-1} z+\frac{87}{16} e^{-1} z^{2}+\frac{29}{16} e^{-1} z^{3}+\frac{29}{64} e^{-1} z^{4}+5 e^{z} \\
& -\frac{216103645}{43046721}-\frac{72555526}{14348907} z-\frac{24666787}{9565938} z^{2}-\frac{4332254}{4782969} z^{3}-\frac{3291049}{1275484} z^{4} \\
& -\frac{114959}{4251528} z^{5}-\frac{257873}{21257640} z^{6}-\frac{456697}{99202320} z^{7}-\frac{24853}{16533720} z^{8}-\frac{338599}{793618560} z^{9} \\
& -\frac{5591}{52907904} z^{10}-\frac{22123}{969978240} z^{11}-\frac{1181}{277136640} z^{12}-\frac{1633}{2401850880} z^{13}-\frac{1254741760}{254} z^{14} \\
& -\frac{107}{11208637440} z^{15}-\frac{1}{1358622720} z^{16}-\frac{1}{30795448320} z^{17} .
\end{aligned}
$$

and so on. 
Now, the solution for the (38) will be the following:

$$
y_{\mathrm{EDM}}(z)=\sum_{j=0}^{2} y_{j}(z)=y_{0}(z)+y_{1}(z)+y_{2}(z)
$$

which converges to the exact solution $y(z)=e^{z}$, as shown in Figure 2.

Table 3 indicates an assessment of the numerical outcomes and the exact solution, and Figure 2 shows that the approximations are very near to the actual values.

\section{Conclusion}

The purpose of this study is to offer an efficient approach for solving higher-order IDEs. This goal was achieved using the Elzaki decomposition method (EDM). The validity and the accuracy of our approach are checked by solving three examples. To explain the technique, plots of the behavior of the solutions are considered to make the shower that the EDM is a very powerful and efficient method to produce the approximate solution.

\section{Data Availability}

The data used to support the findings of this study are available from the corresponding authors upon request.

\section{Conflicts of Interest}

The authors declare that there are no conflicts of interest regarding the publication of this paper.

\section{Acknowledgments}

This work was supported by the Mosul University, College of Computer Sciences and Mathematics, Republic of Iraq.

\section{References}

[1] A. M. Wazwaz, Linear and Nonlinear Integral Equations Methods and Applications, Springer, Berlin, Germany, 2011.

[2] A. M. Wazwaz, A First Course in Integral Equations, World Scientific, New Jersey, NY, USA, 1997.

[3] B. Asady and M. T. Kajani, "Direct method for solving integro differential equations using hybrid Fourier and block-pulse functions," International Journal of Computer Mathematics, vol. 82, no. 7, pp. 889-895, 2005.

[4] H. Danfu and S. Xufeng, "Numerical solution of integrodifferential equations by using CAS wavelet operational matrix of integration," Applied Mathematics and Computation, vol. 194, no. 2, pp. 460-466, 2007.

[5] A. Golbabai and M. Javidi, “Application of He's homotopy perturbation method for nth-order integro-differential equations," Applied Mathematics and Computation, vol. 190, 2007.

[6] A. Karamete and M. Sezer, "A Taylor collocation method for the solution of linear integro-differential equations," International Journal of Computer Mathematics, vol. 79, no. 9, pp. 87-1000, 2002.

[7] K. Maleknejad and F. Mirzaee, "Numerical solution of integro-differential equations by using rationalized Haar functions method," Kybernetes, vol. 35, no. 10, pp. 1735-1744, 2006.

[8] M. T. Kajani, M. Ghasemi, and E. Babolian, "Numerical solution of linear integro-differential equation by using sinecosine wavelets," Applied Mathematics and Computation, vol. 180, no. 2, pp. 569-574, 2006.

[9] W. Wang, "An algorithm for solving the high-order nonlinear Volterra-Fredholm integro-differential equation with mechanization," Applied Mathematics and Computation, vol. 172, no. 1, pp. 1-23, 2006.

[10] S.-Q. Wang and J.-H. He, "Variational iteration method for solving integro-differential equations," Physics Letters A, vol. 367, no. 3, pp. 188-191, 2007.

[11] S. Yalcinbas and M. Sezer, "The approximate solution of highorder linear Volterra-Fredholm integro differential equations in terms of Taylor polynomials," Applied Mathematics and Computation, vol. 112, pp. 291-308, 2000.

[12] S. H. Mohammad and E. S. Al-Rawi, "Solving fractional coupled EW and coupled MEW equations using Bernstein collocation method," Journal of Physics: Conference Series, vol. 1804, no. 1, Article ID 012021, 2021.

[13] J. Zhao and R. M. Corless, "Compact finite difference method for integro-differential equations," Applied Mathematics and Computation, vol. 177, no. 1, pp. 271-288, 2006.

[14] T. M. Elzaki and S. M. Elzaki, "Solution of integro-differential equations by using Elzaki transform," Global Journal of Mathematical Sciences: Theory and Practical, vol. 3, no. 1, pp. 1-11, 2011.

[15] T. M. Elzaki and S. M. Elzaki, "The new integral transform "Tarig transform" properties and applications to differential equations," Elixir International Journal, vol. 38, pp. 42394242, 2011.

[16] T. M. Elzaki, S. M. Elzaki, and E. A. Elnour, "On some applications of new integral transform "Elzaki transform"," Global Journal of Mathematical Sciences: Theory and Practical, vol. 4, no. 1, pp. 15-23, 2011.

[17] P. P. Chopade and S. B. Devi, "Applications of Elzaki transform to ordinary differential and partial differential equations," International Journal of Advanced Research in Computer Science and Software Engineering, vol. 3, no. 5, pp. 38-41, 2015.

[18] A. C. Loyinmim and T. K. Akinfe, "An algorithm for solving the burgers-huxley equation using the Elzaki transform," SN Applied Sciences, vol. 2, no. 1, pp. 1-17, 2020.

[19] M. Naeem, O. F. Azhar, A. M. Zidan, K. Nonlaopon, and R. Shah, "Numerical analysis of fractional-order parabolic equations via Elzaki transform," Journal of Function Spaces, vol. 2021, Article ID 3484482, 10 pages, 2021.

[20] E. Rama, "Application of Elzaki transform to vibrations in mechanical system," Advances in Mathematics: Scientific Journal, vol. 10, no. 4, pp. 1987-1996, 2021.

[21] T. M. Elzaki, "The new integral transform "Elzaki Transform," Global Journal of Pure and Applied Mathematics, vol. 1, pp. 57-64, 2011.

[22] T. M. Elzaki, S. M. Ezaki, and E. A. Elnour, "On the new integral transform "Elzaki transform" fundamental properties investigation and applications," Global Journal of Mathematical Sciences: Theory and Practical, vol. 4, no. 1, pp. 1-13, 2012.

[23] T. M. Elzaki and S. M. Ezaki, "On the solution of integrodifferential equation systems by using ELzaki transform," Global Journal of Mathematical Sciences: Theory and Practical, vol. 3, no. 1, pp. 13-23, 2011.

[24] A. M. Wazwaz, "Anew algorithm for calculating Adomian polynomials for nonlinear operators," Applied Mathematics and Computation, vol. 111, pp. 53-69, 2000. 